\title{
Emerging diagnostic and predictive utilities of natriuretic peptides in diabetes mellitus patients at high cardiovascular risk
}

\author{
Alexander E Berezin ${ }^{* *}$ and Alexander A Berezin ${ }^{2}$ \\ ${ }^{1}$ Internal Medicine Department, State Medical University, Ministry of Health of Ukraine, Zaporozhye, 69035, Ukraine \\ ${ }^{2}$ Internal Medicine Department, Medical Academy of Post-Graduate Education, Ministry of Health of Ukraine, Ukraine²University of Rio Verde, UniRV, Medical \\ School, Rio Verde, GO, Brazil
}

\begin{abstract}
Pre-diabetes and diabetes mellitus (DM) are established cardiovascular (CV) risk factors, which contribute to heart failure (HF) with reduced (HFrEF) and preserved ( $\mathrm{HFpEF}$ ) ejection fraction. Natriuretic peptides (NPs) were found to be useful tool for CV risk stratification among patients with pre-diabetes and T2DM regardless of HF. Previous clinical studies have shown that elevated levels of NPs predicted all-cause and CV mortality, risk of HF manifestation and progression, as well as risk re-admission due to HF. The discriminative potency of NPs for CV death and HF-related events in pre-diabetes and T2DM populations has not been demonstrated beyond traditional CV risk factors. The aim of the review is to accumulate knowledge regarding differential prognostic role of circulating NPs in patients with prediabetes and established T2DM. Presences of HFrEF or HFpEF in T2DM patients may require modification of NP cut-off points to primary diagnose HF and determine HF-related risks. There are several controversies between clinical outcomes and dynamic of circulating levels of NPs in diabetics treated with glucagon-like peptide- 1 agonists and sodium-glucose co-transporter- 2 inhibitors that requires to be elucidated in large clinical studies in the future.
\end{abstract}

\section{Introduction}

Diabetes mellitus (DM) has reached epidemic level and nowadays became the most common metabolic disorders worldwide occupying the $8^{\text {th }}$ leading cause of death [1]. The global statistics of DM has yielded about 382 million people had this disease in 2013 worldwide and by 2030 the number of diabetics will have reached 500 million people [2]. According to the REACH (Reduction of Atherothrombosis for Continued Health) registry patients with type 2 DM (T2DM) compared with patients without DM had higher risk of cardiovascular (CV) death, nonfatal myocardial infarction, or nonfatal stroke [3]. Therefore, T2DM was independently associated with a $33 \%$ greater risk of hospitalization for heart failure (HF), HF-related and CV death $[4,5]$. Noted, T2DM and $\mathrm{CV}$ diseases coexist frequently and $\mathrm{CV}$ risk factors influence significant impact on manifestation and progression of both conditions [6]. Although CV factors are affected by several antihyperglycemic medications, there is not complete correspondence between a control for conventional CV risk factors including glycemic status by life style modification, drug prescription and diminishing risk of T2DMrelated and CV complications [7-9]. In this context, risk stratification strategy among patients with prediabetes and known T2DM requires to be personally modified and this improvement perhaps can be based on biomarker prediction scores [10]. Among numerous circulating biomarkers and multiple biomarker-based models reflecting various stages of $\mathrm{CV}$ disease manifestation and $\mathrm{HF}$ advance natriuretic peptides (NPs) continue to be a core element in CV risk assessment and targets in guided therapy of HF [11]. However, serious variability of circulating levels of NPs in patients with metabolic diseases, including abdominal obesity, metabolic syndrome and DM, due to several reasons requires an adjustment of the diagnostic and predictive cut-off levels of NPs, while the NPs remain useful diagnostic and predictive biomarker for all-cause mortality, CV death and HF regardless of presence of HF. The aim of the mini review is to accumulate knowledge regarding differential prognostic role of circulating NPs in patients with prediabetes and established T2DM.

\section{Myocardial biomechanical stress in T2DM}

Previous magnetic resonance imaging studies have revealed that alterations in glucose metabolism were independently associated with left ventricular (LV) concentric remodeling, less spherical shape, and reduced systolic myocardial shortening in the general population [12-14]. Moreover, speckle-tracking echocardiography studies have yielded that myocardial shortening, LV torsion and myocardial strain was progressively decreased with higher HOMA-IR and torsion was increased only with less severe insulin resistance in individuals with prediabetes $[15,16]$. In the CARDIA (Coronary Artery Risk Development in Young Adults) study patients with established T2DM had lowered LV ejection fraction (LVEF), longitudinal systolic strain, and early diastolic strain rate when compared with patients having

${ }^{\star}$ Correspondence to: Alexander E. Berezin, Professor, $\mathrm{MD}, \mathrm{PhD}$, Senior Consultant of Therapeutic Unit, Internal Medicine Department, State Medical University for Zaporozhye, 26, Mayakovsky av., Zaporozhye, Postcode 69035, Ukraine, Tel: +38 061 2894585, Fax: +38 0612894585, E-mail: aeberezin@gmail.com

Key words: type 2 diabetes mellitus, prediabetes, cardiovascular risk, biomarkers, natriuretic peptides

Received: February 08, 2020; Accepted: February 21, 2020; Published: February 24,2020 
normal glucose metabolism [16]. The population STAAB cohort study has demonstrated that LV global longitudinal strain and torsion were inversely associated with glycosylated hemoglobin (HbA1c) and insulin resistance and that these parameters were found significant lowered in diabetics in compared with non-diabetics without known CV disease [17].

In fact, glucose abnormalities, lipid toxicity, altered tissue reparation, accelerating atherosclerosis, and co-existing conventional CV risk factors are causes to develop diabetic cardiomyopathy as well as HF related to ischemia causes $[18,19]$. In fact, atherosclerosis, systemic and micro vascular inflammation, myocardial fibrosis, myocardial infarction, or LV contractile / diastolic dysfunction due to microvascular obstruction are primary reasons for myocardial remodeling, for which myocardial biomechanical stress is discussed as crucial pathogenetic mechanism leading to HF with preserved ejection fraction (HFpEF) and reduced ejection fraction (HFrEF) [20]. Indeed, T2DM through several molecular mechanisms, such as renin-angiotensin system activation, cardiac autonomic neuropathy, alterations in calcium homeostasis, generation of reactive oxygen or nitrogen species leading to mitochondrial dysfunction, causes ischemic myocardial injury, which represents the major cause of death in diabetic subjects, diabetic cardiomyopathy summarizes adverse effects of T2DM on the myocardium regardless of presence of coronary artery disease (CAD) and hypertension [21]. Having evidence that conventional CV risk factors, $\mathrm{CAD}$, and diabetic cardiomyopathy influence negatively on mortality rate and quality-of-life among T2DM patients, there is a suggestion that cardiac biomarkers reflecting various faces of altered cardiac remodeling and HF advance, such as natriuretic peptides, would ensure add-on incremental value for the prediction of clinical outcomes (death, MACEs, hospital admission, HF-related events) in the patient population. Moreover, the levels of natriuretic peptides, could demonstrate personifying predictive information that would be able to yield the greatest predictive potency beyond conventional $\mathrm{CV}$ risk factors [22].

\section{Natriuretic peptides: biological role and function}

Biological role of NPs' system as a core element in a regulation of natriuresis, vasodilation, water and sodium homeostasis are well established and wide known [23]. Indeed, predominantly atrial (ANP) and brain (BNP) and rarely C-type of NP are embedded onto a regulation of cardiorenal homeostasis through appropriate receptor A (NPRA). Clearance of NPs are mediated by their proteolysis by endogenous endopeptidase called neprilysin that activates physiological pathways by which NPs are effectively removed from circulation through receptor-mediated internalization by the NP receptor C (NPRC) [24]. In fact, both types of the NPs' receptors (NPRA and NPRC) as well as activity and circulating levels of neprilysin ensure determines the NP bioactivity [25]. The main triggers for NPs' secretion are myocardial stretching, fluid overload, ischemia / hypoxia, inflammation, and renin-angiotensin-aldosterone system (RAAS) [26]. Although system of the NPs is a physiological antagonist of RAAS, there is a wide range of evidence regarding that the NPs through adipose tissue-expressed NPRA and NPRC reciprocally regulate lipolytic activity of adipocytes similar to catecholamine-derived effect that is mediated by the $\beta$ adrenergic receptors [27]. Additionally, NPs via p38 MAP kinase act as a triggers for over-expression of brown fat genes to increase energy expenditure and regulate adaptive thermogenesis [28]. Therefore, in human cells, including adipocytes, muscle cell, hepatocytes, NPs promote transcriptional regulation of genes involved in mitochondrial biogenesis, uncoupled respiration (peroxisome proliferator-activated receptor- $\gamma$ coactivator- $1 \alpha$ and uncoupling protein 1$)$, lipid oxidation, as well as glucose tolerance insulin resistance [29,30]. Overall, activation of NPRA signaling pathway in skeletal muscle and hepatocytes is crucial for the maintenance of long-term insulin sensitivity and this phenomenon can link transformation of pre-diabetes to T2DM as well as ensures rockets of $\mathrm{CV}$ risk [31,32].

\section{Natriuretic peptides in pre-diabetes and T2DM}

Previous studies have shown that patients with abdominal obesity, metabolic syndrome, and T2DM may have rather lover levels of NPs than healthy volunteers and that this effect relates to clearance of NPs especially activity of neprilysin $[33,34]$. Indeed, insulin may upregulate the NPRC expression in the subcutaneous fat depot in obese individuals [35], while the difference between healthy volunteers and obese patients in the circulating levels of NP's was not confirmed by several investigators $[36,37]$. Therefore, kidney clearance of NPs was found to be worse in T2DM with nephropathy that was associated with increased circulating levels of BNP and NT-pro-BNP [38]. However, the reasons for NP level fluctuation in patents with metabolic disease remain still uncertain.

\section{Natriuretic peptides in HF associated with abnormalities of glucose status}

Current clinical guidelines recommend using biomarker level measure to diagnose HF when diagnosis is uncertain, stratify patients from general population into group with higher risk of $\mathrm{CV}$ mortality rate and HF manifestation, as well as to prognosticate risks of HF advance and 60-day re-admission regardless of presentation of abdominal obesity, pre-diabetes, and T2DM [39,40]. However, asymptomatic patients form general population to be stratified at a risk of death and HF onset should have higher circulating levels of NTproBNP $(>300 \mathrm{pg} / \mathrm{mL})$ than individuals having signs and symptoms of HF (125 pg/mL) [40]. In fact, increased age requires re-checking diagnostic NT-proBNP cut-off point for patients suspecting cardiac dysfunction [38,41]. Interestingly, among patients without T2DM, elevated levels of NPs yielded greater predictive accuracy for CAD, MACEs, CV mortality and HF manifestation than in T2DM patients $[39,42]$. Although the circulation levels of NT-proBNP were not differ between male and female in general population, elevated NT-proBNP concentrations rather conferred a higher risk of $\mathrm{CV}$ mortality in women with HFpEF than in male with HFpEF [43]. However, T2DM patients have demonstrated more LV hypertrophy and adverse cardiac remodeling, but systolic and diastolic LV function parameters as well as NT-proBNP serum levels were found to be similar in T2DM and non-T2DM patients [44]. Therefore, in patients with abdominal obesity, metabolic syndrome and T2DM serum levels of NPs were found to be independent predictors for atherosclerosis, albuminuria, atrial fibrillation, pulmonary hypertension, and sudden death rate, but in patient population with HFrEF predictive value of NPs for these outcomes was not related to presentation of prediabetes and T2DM [45-47]. Despite NT-proBNP levels were lower in overweight/ obesity patients, even in those with T2DM, but higher than in healthy volunteers, NT-proBNP level may have predictive value to diagnose cardiac abnormalities regardless of glucose status [48]. Moreover, multiple biomarkers' models including NP levels occurred to be more prognostically accurate for HF onset in non-T2DM patients that in prediabetics with abdominal obesity and T2DM patients $[49,50]$.

There are several controversies regarding predictive abilities of elevated levels of NPs in patients with metabolically healthy obesity and metabolic syndrome. First controversy was related to evidence that NPs 
were not better than conventional cardiac biomarkers, such as cardiac troponins, soluble ST2, ischemia-modified albumin in prediction of the onset of future microvascular and macrovascular complications in obese/ prediabetes patients without known CV disease [51]. In contrast, metabolic biomarkers (adiponectin, chemerin, visfatin) sufficiently increased predictive value of NT-proBNP levels for MACEs in patients with various glucose statuses and established HF [52-54]. The next controversy affects negative associations between NPs' serum levels (including ANP, NT-proANP, NT-proBNP) and several components of the metabolic syndrome in young people without CV disease, while the inverse relationships between several components of the metabolic syndrome and circulating NP concentrations were found in middleaged and elderly populations [55]. Finally, elevated levels of NTproBNP remain strong predictor of death in patients with established $\mathrm{CV}$ diseases including CAD and HF, even with the confounding effect of pre-diabetes and T2DM [56], but it is still uncertain whether the discriminative potency of NPs for CV death and events in prediabetes and T2DM populations beyond CV disease would be independent from traditional CV risk factors.

\section{Controversial prognostication abilities of natriuretic peptides in clinical trials among T2DM patients}

Recent clinical trials have shown that improving metabolic status in HFrEF patients with pre-diabetes and T2DM with glucagon-like peptide-1 [GLP-1] analogue (liraglutide) [57], sodium-glucose cotransporter-2 [SGLT2] (empagliflozine) [58-60], was associated with a tendency to NT-proBNP serum level decrease as a secondary surrogate end point, cardiac protective effect, and beneficial CV outcomes. In contrast, among patients with prediabetes and T2DM without HF serum levels of NT-proBNP remained unaltered regardless of improving glucose homeostasis and decreased CV risk [61]. Interestingly, the change in NT-proBNP serum levels correlated negatively with baseline NT-proBNP levels in T2DM [62]. Additionally, in the DEFINE-HF Trial SGLT2 dapagliflozin did not affect mean NTproBNP serum levels, but increased the proportion of patients (as diabetics, as well as non-diabetics) experiencing clinically meaningful improvements in HFrEF-related clinical status [63]. It is difficult to speculate a plausible mechanistic reason why GLP-1 analogues and SGLT2 have demonstrated controversial effect on NP circulating levels in patients with T2DM having HF or without it. Interestingly, dipeptidyl peptidase (DPP)-4 inhibitors had been reported to have rather neutral effect than deteriorating impact on myocardium in preclinical studies and early large-scale trials [64-66]. However, on the one hand DPP4 inhibitors may increase the ability of GLP-1 to stimulate cyclic adenosine monophosphate in cardiac myocytes, and on the other hand they potentiate the effects of stromal cell-derived factor-1 aggravating cardiac fibrosis and indirectly increase in circulating levels of NPs [67]. Finally, an increased risk of HF progression appeared to be a class effect of DPP-4 inhibitors, even in patients without a history of HF [68]. However, other antidiabetic drugs, i.e. metformin, thiazolidinediones, have demonstrated predictably clear impact on circulating levels of NP, i.e. metformin did not increase the concentration of ones, but thiazolidinediones through fluid retention acted as triggers for NP level augment [68].

\section{Conclusions}

Natriuretic peptides are useful tool for CV risk stratification among patients with pre-diabetes and T2DM regardless of HF. Presences of HFrEF or HFpEF in T2DM patients may require modification of NP cut-off points to primary diagnose HF and determine HF-related risks. There are several controversies between clinical outcomes and dynamic of circulating levels of NPs in diabetics treated with GLP-1 agonists and SGLT2 inhibitors that requires to be elucidated in large clinical studies in the future.

\section{Funding and grants}

This research received no specific grant from any funding agency in the public, commercial, or not-for-profit sectors.

\section{Conflict of interests}

Authors have no conflict of interest.

\section{References}

1. Tao Z, Shi A, Zhao J (2015) Epidemiological perspectives of diabetes. Cell Biochem Biophys 73: 181-185.

2. Benjamin EJ, Virani SS, Callaway CW, Chamberlain AM, Chang AR, et al. (2018) Heart disease and stroke statistics-2018 update: A report from the american heart association. Circulation 137: e67-67e492. [Crossref]

3. Cavender MA, Steg PG, Smith SC, Eagle K, Ohman EM, et al. (2015) Reach registry investigators. impact of diabetes mellitus on hospitalization for heart failure, cardiovascular events, and death: Outcomes at 4 years from the reduction of atherothrombosis for continued health. Circulation 132: 923-931.

4. Selvin E, Parrinello CM, Sacks DB, Coresh J (2014) Trends in prevalence and control of diabetes in the United States, 1988-1994 and 1999-2010. Ann Intern Med 160: $517-$ 525. [Crossref]

5. Bae JC, Cho NH, Suh S, Kim JH, Hur KY, et al. (2015) Cardiovascular disease incidence, mortality and case fatality related to diabetes and metabolic syndrome: a community-based prospective study (Ansung-Ansan cohort 2001-12). J Diabetes 7: 791-799.

6. Strain WD, Paldinius PM (2018) Diabetes, cardiovascular disease and the microcirculation. Cardiovasc Diabetol 17: 50-57.

7. Alvarez CA, Lingvay I, Vuylsteke V, Koffarnus RL, McGuire DK (2015) Cardiovascular risk in diabetes mellitus: Complication of the disease or of antihyperglycemic medications. Clin Pharmacol Ther 98: 145-161.

8. Komaru Y, Takeuchi T, Suzuki L, Asano T, Urayama KY (2019) Recurrent cardiovascular events in patients with newly diagnosed acute coronary syndrome: Influence of diabetes and its management with medication. $J$ Diabetes Complications 107511.

9. Yang YS, Yang BR, Kim MS, Hwang Y, Choi SH (2020) Low-density lipoprotein cholesterol goal attainment rates in high-risk patients with cardiovascular diseases and diabetes mellitus in Korea: a retrospective cohort study. Lipids Health Dis 19: 1-5.

10. Berezin AE (2019) Prognostication of clinical outcomes in diabetes mellitus: Emerging role of cardiac biomarkers. Diabetes Metab Syndr 13: 995-1003.

11. Berezin AE (2017) Cardiac biomarkers in diabetes mellitus: New dawn for risk stratification? Diabetes Metab Syndr 11: S201-S208.

12. Yoneyama K, Venkatesh BA, Wu CO, Mewton N, Gjesdal O, et al. (2018) Diabetes mellitus and insulin resistance associate with left ventricular shape and torsion by cardiovascular magnetic resonance imaging in asymptomatic individuals from the multi-ethnic study of atherosclerosis. J Cardiovasc Magn Reson 20: 50-53.

13. Young AA, Cowan BR (2012) Evaluation of left ventricular torsion by cardiovascular magnetic resonance. J Cardiovasc Magn Reson 1: 14-49.

14. Ilercil A, Devereux RB, Roman MJ, Paranicas M, O'grady MJ, et al. (2001) Relationship of impaired glucose tolerance to left ventricular structure and function: the strong heart study. Am Heart J 141: 992-998.

15. Lin JL, Sung KT, Su CH, Chou TH, Lo CI, et al. (2018) Cardiac structural remodeling, longitudinal systolic strain, and torsional mechanics in lean and nonlean dysglycemic chinese adults. Circ Cardiovasc Imaging 11: e007047.

16. Kishi S, Gidding SS, Reis JP, Colangelo LA, Venkatesh BA, et al. (2017) Association of Insulin Resistance and Glycemic Metabolic Abnormalities with LV structure and function in middle age: The CARDIA Study. JACC Cardiovasc Imaging 10: 105-114.

17. Morbach C, Walter BN, Breunig M, Liu D, Tiffe T, et al. (2019) STAAB consortium. Speckle tracking derived reference values of myocardial deformation and impact of cardiovascular risk factors - Results from the population-based STAAB cohort study. PLoS One 14: e0221888. 
18. Shah RV, Abbasi SA, Heydari B, Rickers C, Jacobs DR, et al. (2013) Insulin resistance, subclinical left ventricular remodeling, and the obesity paradox: MESA (multi-ethnic study of atherosclerosis). J Am Coll Cardiol 61: 1698-1706.

19. Turkbey EB, McClelland RL, Kronmal RA, Burke GL, Bild DE, et al. (2010) The impact of obesity on the left ventricle: The multi-ethnic study of atherosclerosis (MESA). JACC Cardiovasc Imaging 3: 266-74.

20. Bugger H, Abel ED (2014) Molecular mechanisms of diabetic cardiomyopathy. Diabetologia 57: 660-671.

21. Hölscher ME, Bode C, Bugger H (2016) Diabetic Cardiomyopathy: Does the Type of Diabetes Matter? Int J Mol Sci 17. [Crossref]

22. Tarquini R, Lazzeri C, Pala L, Rotella CM, Gensini GF (2011) The diabetic cardiomyopathy. Acta Diabetol 48: 173-181. [Crossref]

23. Potter LR, Abbey-Hosch S, Dickey DM (2006) Natriuretic peptides, their receptors, and cyclic guanosine monophosphate-dependent signaling functions. Endocr Rev 27: 47-72. [Crossref]

24. Potter LR, Hunter T (2001) Guanylyl cyclase-linked natriuretic peptide receptors: structure and regulation. $J$ Biol Chem 276: 605-6060

25. Kovacova Z, Tharp WG, Liu D, Wei W, Xie H, et al. (2016) Adipose tissue natriuretic peptide receptor expression is related to insulin sensitivity in obesity and diabetes. Obesity 24: 820-828.

26. Moro C (2016) Targeting cardiac natriuretic peptides in the therapy of diabetes and obesity. Expert Opin Ther Targets 20: 1445-1452. [Crossref]

27. Bordicchia M, Ceresiani M, Pavani M, Minardi D, Polito M, et al. (2016) Insulin/ glucose induces natriuretic peptide clearance receptor in human adipocytes: a metabolic link with the cardiac natriuretic pathway. Am J Physiol Regul Integr Comp Physiol 311: 104-114.

28. Bordicchia M, Liu D, Amri EZ, Ailhaud G, Dess Fulgheri P (2012) Cardiac natriuretic peptides act via 38 MAPK to induce the brown fat thermogenic program in mouse and human adipocytes. J Clin Invest 122: 1022-1036.

29. Sengenes C, Berlan M, De Glisezinski I, Lafontan M, Galitzky J (2000) Natriuretic peptides: a new lipolytic pathway in human adipocytes. FASEB J 14: 1345-1351

30. Engeli S, Birkenfeld AL, Badin PM, Bourlier V, Louche K, et al. (2012) Natriuretic peptides enhance the oxidative capacity of human skeletal muscle. J Clin Invest 122: 4675-4679

31. Coue M, Badin PM, Vila IK, Laurens C, Louche K, et al. (2015) Defective Natriuretic Peptide Receptor Signaling in Skeletal Muscle Links Obesity to Type 2 Diabetes. Diabetes 64: 4033-4045.

32. Højlund K (2014) Metabolism and insulin signaling in common metabolic disorders and inherited insulin resistance. Dan Med J 61: B4890. [Crossref]

33. Chen Y, Burnett JC (2017) Biochemistry, therapeutics, and biomarker implications of neprilysin in cardiorenal disease. Clin Chem 63: 108-115.

34. Lee KM, Lee MC, Lee CJ, Chen YC, Hsu BG (2018) Inverse association of n-terminal proâ€'b-type natriuretic peptide level with metabolic syndrome in kidney transplant patients. Transplant Proc 50: 2496-2501.

35. Pivovarova O, Gogebakan O, Kloting N, Sparwasser A, Weickert MO, et al. (2012) Insulin up-regulates natriuretic peptide clearance receptor expression in the subcutaneous fat depot in obese subjects: a missing link between CVD risk and obesity? J Clin Endocrinol Metab 97: 731-739

36. Sarzani R, Salvi F, Dessi Fulgheri P, Rappelli A (2008) Renin-angiotensin system, natriuretic peptides, obesity, metabolic syndrome, and hypertension: an integrated view in humans. J Hypertens 26: 831-843

37. Ahued-Ortega JA, Leon-Garcia PE, Hernandez-Perez E (2018) Correlation of plasma B-type natriuretic peptide levels with metabolic risk markers. Med Clin 151: 481-486.

38. Di Marca S, Rando A, Cataudella E, Pulvirenti A, Alaimo S, et al. (2018) B-type natriuretic peptide may predict prognosis in older adults admitted with a diagnosis other than heart failure. Nutr Metab Cardiovasc Dis 28: 636-642.

39. Wong YK, Cheung CYY, Tang CS, Hai JSH, Lee CH, et al. (2019) High-sensitivity troponin I and B-type natriuretic peptide biomarkers for prediction of cardiovascular events in patients with coronary artery disease with and without diabetes mellitus. Cardiovasc Diabetol 18: 171.

40. Ponikowski P, Voors AA, Anker SD, Bueno H, Cleland JGF, et al. (2016) ESC Scientific Document Group. 2016 ESC guidelines for the diagnosis and treatment of acute and chronic heart failure: the Task Force for the diagnosis and treatment of acute and chronic heart failure of the European Society of Cardiology (ESC) developed with the special contribution of the Heart Failure Association (HFA) of the ESC. Eur Heart J37: 2129-2200.
41. Yancy CW, Jessup M, Bozkurt B, Butler J, Casey DE, et al. (2017) ACC/AHA/ HFSA focused update of the $2013 \mathrm{ACCF} / \mathrm{AHA}$ guideline for the management of heart failure: a report of the American College of Cardiology/American Heart Association Task Force on Clinical Practice Guidelines and the Heart Failure Society of America. Circulation 136: e137-e161.

42. Benomar K, Espiard S, Loyer C, Jannin A, Vantyghem MC (2018) Atrial natriuretic hormones and metabolic syndrome: recent advances. Presse Med 47: 116-124.

43. Palau P, Bertomeu-Gonzalez V, Sanchis J, Soler M, de la Espriella R, et al. (2019) Differential prognostic impact of type 2 diabetes mellitus in women and men with heart failure with preserved ejection fraction. Rev Esp Cardiol 19: 264-266.

44. Lindman BR, Davila-Roman VG, Mann DL, McNulty S, Semigran MJ, et al. (2014) Cardiovascular phenotype in HFpEF patients with or without diabetes: a RELAX trial ancillary study. J Am Coll Cardiol 64: 541-549.

45. Georgakopoulos C, Vlachopoulos C, Lazaros G, Tousoulis D (2019) Biomarkers of Atrial Fibrillation in Metabolic Syndrome. Curr Med Chem 26: 898-908. [Crossref]

46. Obaid N, Hadidy SE, Badry ME, Khaled H (2019) The Outcome of Diabetic Patients with Cardiomyopathy in Critical Care Unit: Hospital and Short-Term Outcome in a Period of Six Months to One Year. Open Access Maced J Med Sci 7: 2796-2801.

47. Horwich TB, Hamilton MA, Fonarow GC (2006) B-type natriuretic peptide levels in obese patients with advanced heart failure. $J$ Am Coll Cardiol 47: 85-90

48. Krzesinski P, Uzieblo zyczkowska B, Gielerak G, Stanczyk A, Piotrowicz K, et al (2017) Echocardiographic assessment and N-terminal pro-brain natriuretic peptide in hypertensives with metabolic syndrome. Adv Clin Exp Med 26: 295-301.

49. Mocan M, Anton F, Suciu S, Rahaian R, Blaga SN, Farcas AD (2017) Multimarker assessment of diastolic dysfunction in metabolic syndrome patients. Metab Syndr Relat Disord 15: 507-514.

50. Prickett TCR, Darlow BA, Troughton RW, Cameron VA, Elliott JM, et al. (2018) New Insights into cardiac and vascular natriuretic peptides: findings from young adults born with very low birth weight. Clin Chem 64: 363-373.

51. Parsanathan R, Jain SK (2019) Novel invasive and noninvasive cardiac-specific biomarkers in obesity and cardiovascular diseases. Metab Syndr Relat Disord 10: 1-10

52. Zhou X, Tao Y, Chen Y, Xu W, Qian Z, et al. (2019) Serum Chemerin as a Novel Prognostic Indicator in Chronic Heart Failure. J Am Heart Assoc 8: e012091

53. Contaifer D, Buckley LF, Wohlford G, Kumar NG, Morriss JM, et al. (2019) Metabolic modulation predicts heart failure tests performance. PLoS One 14(6): e218153.

54. Berezin AE, Samura TA, Kremzer AA, Berezina TA, Martovitskaya YV, et al. (2016) An association of serum visfatin level and number of circulating endothelial progenitor cells in type 2 diabetes mellitus patients. Diabetes Metab Syndr 10: 205-212.

55. Goharian TS, Goetze JP, Faber J, Andersen LB, Jeppesen JL, et al. (2017) Associations of pro-atrial natriuretic peptide with components of the metabolic syndrome in adolescents and young adults from the general population. Am J Hypertens 30: 561568

56. Huang FY, Peng Y, Deng XX, Huang BT, Xia TL, et al. (2017) The influence of metabolic syndrome and diabetes mellitus on the N-terminal pro-B-type natriuretic peptide level and its prognostic performance in patients with coronary artery disease. Coron Artery Dis 28: 159-165.

57. Nomoto H, Miyoshi H, Furumoto T, Oba K, Tsutsui H, et al. (2015) SAIS study group a comparison of the effects of the GLP-1 analogue liraglutide and insulin glargine on endothelial function and metabolic parameters: A randomized, controlled trial sapporo athero-incretin study 2 (SAIS2). PLoS One 10: e0135854.

58. Verma S, Mazer CD, Yan AT, Mason T, Garg V, et al. (2019) Effect of empagliflozin on left ventricular mass in patients with type 2 diabetes mellitus and coronary artery disease: The EMPA-Heart cardiolink-6 randomized clinical trial. Circulation 140: 1693-1702.

59. Jensen J, Omar M, Kistorp C, Poulsen MK, Tuxen C, et al. (2019) Empagliflozin in heart failure patients with reduced ejection fraction: a randomized clinical trial (Empire HF). Trials 20: 370-374.

60. Ferrannini E, Baldi S, Frascerra S, Astiarraga B, Barsotti E, et al. (2017) Renal handling of ketones in response to sodium-glucose cotransporter 2 inhibition in patients with type 2 diabetes. Diabetes Care 40: 771-776.

61. Cho KY, Nakamura A, Omori K, Takase T, Miya A, et al. (2019) Effect of switching from pioglitazone to the sodium glucose co-transporter-2 inhibitor dapagliflozin on body weight and metabolism-related factors in patients with type 2 diabetes mellitus: An open-label, prospective, randomized, parallel-group comparison trial. Diabetes Obes Metab 21: 710-714. 
62. Nassif ME, Windsor SL, Tang F, Khariton Y, Husain M, et al. (2019) Dapagliflozin effects on biomarkers, symptoms, and functional status in patients with heart failure with reduced ejection fraction: The define-hf Trial. Circulation 140: 1463-1476.

63. Hiemstra JA, Lee DI, Chakir K, Marshall KD, Zgoda PJ, et al. (2016) Saxagliptin and tadalafil differentially alter cyclic guanosine monophosphate (cGMP) signaling and left ventricular function in aortic-banded mini-swine. J Am Heart Assoc 5: e003277.

64. Scirica BM, Braunwald E, Raz I, Cavender MA, Morrow DA, et al. (2014) SAVORTIMI 53 steering committee and investigators. heart failure, saxagliptin, and diabetes mellitus: observations from the SAVOR-TIMI 53 randomized trial. Circulation 130: $1579-1588$.
65. Li L, Li S, Deng K, Liu J, Vandvik PO, et al. (2016) Dipeptidyl peptidase-4 inhibitors and risk of heart failure in type 2 diabetes: systematic review and meta-analysis of randomised and observational studies. BMJ 352: i610.

66. Packer M (2018) Worsening heart failure during the use of DPP-4 inhibitors: Pathophysiological mechanisms, clinical risks, and potential influence of concomitant antidiabetic medications. JACC Heart Fail 6: 445-451.

67. Kappel BA, Marx N, Federici M (2015) Oral hypoglycemic agents and the heart failure conundrum: Lessons from and for outcome trials. Nutr Metab Cardiovasc Dis 25: 697705 .

Copyright: $(2020$ Berezin AE. This is an open-access article distributed under the terms of the Creative Commons Attribution License, which permits unrestricted use, distribution, and reproduction in any medium, provided the original author and source are credited. 